\title{
INSTANTONS AND QUATERNIONS
}

\section{Stefan Vandoren}

YITP, SUNY at Stony Brook, Stony Brook, NY 11794-3840, USA

E-mail: 'vandoren@insti.physics. sunysb.edu'

Abstract: We relate the moduli space of Yang-Mills instantons to quaternionic manifolds. For instanton number one, the Wolf spaces play an important role. We apply these ideas to instanton calculations in $\mathcal{N}=4 \mathrm{SYM}$ theory.

Instantons in Yang-Mills theories are defined by the solutions to the self-duality equation in four-dimensional Euclidean space,

$$
F_{\mu \nu}={ }^{*} F_{\mu \nu}=\frac{1}{2} \epsilon_{\mu \nu \rho \sigma} F_{\rho \sigma},
$$

and are characterized by an integer number, the topological charge,

$$
k=-\frac{1}{16 \pi^{2}} \int \mathrm{d}^{4} x \operatorname{Tr} F_{\mu \nu}{ }^{*} F_{\mu \nu} .
$$

For $k=1$, one can explicitly solve these equations $[\overline{1} 1, \overline{2}, \overline{2}$, and for gauge group $S U(2)$, the instanton gauge field one-form $A$ and its field strength $F$ can be elegantly written in terms of quaternions $x=x_{\mu} \sigma_{\mu}$, with $\sigma_{\mu}=(\vec{\tau}, i)$, [해]

$$
\begin{aligned}
A & =i \operatorname{Im} \frac{\left(\bar{x}-\bar{x}_{0}\right) \mathrm{d} x}{\rho^{2}+\left|x-x_{0}\right|^{2}} \\
F & =\frac{2 \rho^{2} \mathrm{~d} \bar{x} \wedge \mathrm{d} x}{\left(\rho^{2}+\left|x-x_{0}\right|^{2}\right)^{2}},
\end{aligned}
$$

where the norm of quaternions is defined as $\left|x^{2}\right|=$ $\frac{1}{2} \operatorname{tr}(x \bar{x})=x_{\mu} x_{\mu}$. This solution has five collective coordinates, four positions $x_{0}^{\mu}$ and a size $\rho$, but one can act with rigid $S U(2)$ gauge rotations with angles $\vec{\theta}$ to generate new inequivalent solutions. So in this example, there are eight collective coordinates.

The purpose of this letter is to elaborate on the geometry of the moduli space of collective coordinates, and to apply this knowledge to instanton calculations in $\mathcal{N}=4$ supersymmetric Yang-Mills theories.

For $k=1$ and gauge group $S U(2)$, the moduli space is simply $\mathbf{R}^{8}$ (in fact, it is $\mathbf{R}^{4} \times \mathbf{R}^{4} / Z_{2}$
, which has an orbifold singularity corresponding to zero size instantons), with metric

$$
\mathrm{d} s^{2}=\mathrm{d} x_{0}^{\mu} \mathrm{d} x_{0}^{\mu}+(\mathrm{d} \rho)^{2}+\rho^{2} \mathrm{~d} S^{3} .
$$

This metric can be computed by evaluating the inner product of zero modes, which are the derivatives of the instanton field with respect to the collective coordinates, as was demonstrated in

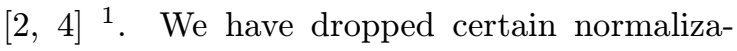
tion factors, which are irrelevant for the present discussion. The last term in $\left(\begin{array}{c}\overline{4} \\ -1\end{array}\right)$ comes from the gauge orientation zero modes, whose corresponding collective coordinates $\vec{\theta}$ parametrize $S U(2) \equiv$ $S^{3}$.

For $k=1$ and gauge group $S U(N)$, one can construct all instanton solutions by embedding the $S U(2)$ instanton inside $S U(N)$, and acting with rigid gauge transformations on the chosen embedding,

$$
\begin{aligned}
A_{\mu}^{S U(N)} & =U(\phi)\left(\begin{array}{cc}
0 & 0 \\
0 & A_{\mu}^{S U(2)}
\end{array}\right) U^{\dagger}(\phi) \\
U & \in \frac{S U(N)}{S U(N-2) \times U(1)} .
\end{aligned}
$$

Notice that we have divided by the stability group of the instanton embedding. The $S U(N-2)$ fac-

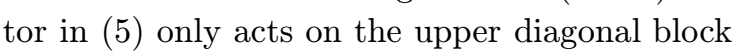
and commutes with the $S U(2)$ embedding. Furthermore, there is an extra singlet, corresponding to the $U(1)$ factor in the stability group, commuting with $S U(2)$ (e.g., for $S U(3)$ with the stan-

\footnotetext{
${ }^{1}$ For a recent review on instantons, and how to determine the zero modes and metric, see e.g. [5]
} 
dard Gell-Mann matrices, the first three determine the $S U(2)$ embedding, whereas the eighth generator commutes with this embedding and is the $U(1)$ singlet mentioned above).

The dimension of this coset space is $4 N-$ 5 , and we denote the coordinate-angles by $\phi=$ $\left\{\vec{\theta}, q_{i}^{M}\right\}, i=1, \ldots, N-2, M=1, \ldots, 4$ so counting leads to $4 N$ collective coordinates in total, in agreement with standard index theorems.

The metric on the moduli space of $k=1$ $S U(N)$ instantons is [4]

$$
\mathrm{d} s^{2}=\mathrm{d} x_{0}^{\mu} \mathrm{d} x_{0}^{\mu}+(\mathrm{d} \rho)^{2}+\rho^{2} \mathrm{~d} S^{3}+\rho^{2} \mathrm{~d} X_{N-2},
$$

where $\mathrm{d} X_{N-2}$ stands for the metric element on the $4(N-2)$-dimensional space

$$
X_{N-2}=\frac{S U(N)}{S U(N-2) \times S U(2) \times U(1)} .
$$

Actually this metric is the one obtained by working infinitesimally in the angles $\phi$, for which it is known that the $\vec{\theta}$ zero modes are orthogonal to the $q_{i}^{M}$ zero modes, see for instance [5in]. For reasons to be explained below, one expects that the full metric in ( $\overline{6}_{1}^{(\bar{G})}$ is of the form where $S^{3}$ is fibered non-trivially over $X_{N-2}$.

The space $X_{N-2}$ is known as one of the Wolf spaces $[\overline{6}|\bar{b}|$ and is an example of a quaternionic manifold, which we will discuss later. The angles $q_{i}^{M}$ can then be interpreted as $N-2$ quaternions, which coordinatize the quaternionic manifold. Ignoring the center of mass coordinates $x_{0}^{\mu}$, the metric has the form of a cone, with radial parameter $\rho$.

For $k=1$ and gauge group $S O(N)$, there are $4 N-8$ collective coordinates. The one-instanton solution is constructed by choosing an $S U(2)$ inside an $S O(4)=S U(2) \times S U(2)$ embedding in $S O(N)[\overline{7}]$. The stability group of this instanton is $S O(N-4) \times S U(2)$, and performing a similar counting as for the $S U(N)$ case, one indeed obtains $4 N-8$ collective coordinates. The metric on the moduli space is now

$$
\mathrm{d} s^{2}=\mathrm{d} x_{0}^{\mu} \mathrm{d} x_{0}^{\mu}+(\mathrm{d} \rho)^{2}+\rho^{2} \mathrm{~d} S^{3}+\rho^{2} \mathrm{~d} Y_{N-4},
$$

where $\mathrm{d} Y_{N-4}$ stands for the metric element on the $4(N-4)$-dimensional Wolf space

$$
Y_{N-4}=\frac{S O(N)}{S O(N-4) \times S O(4)} .
$$

Finally we analyze the gauge group $S p(N)$. Here we can simply choose the lower diagonal $S U(2)=S p(1)$ embedding inside $S p(N)$. The stability group of this embedding is now $S p(N-$ 1 ), and the moduli space metric is

$$
\mathrm{d} s^{2}=\mathrm{d} x_{0}^{\mu} \mathrm{d} x_{0}^{\mu}+(\mathrm{d} \rho)^{2}+\rho^{2} \mathrm{~d} S^{3}+\rho^{2} \mathrm{~d} H P_{N-1},
$$

with corresponding $4(N-1)$-dimensional quaternionic projective space

$$
H P_{N-1}=\frac{S p(N)}{S p(N-1) \times S p(1)} .
$$

Counting leads to $4(N+1)$ collective coordinates, which agrees again with index theorems. One could do a similar analysis for the exceptional gauge groups, and obtain a corresponding quaternionic manifold. It is known that for any semisimple Lie algebra, there is a corresponding quaternionic Wolf space [íi]. In general a quaternionic manifold (in the mathematics literature called quaternionic-Kähler although they are not of the Kählerian type) is a $4 n$-dimensional Riemannian space with holonomy group contained in $S p(1) \times$ $S p(n)$. These spaces are always Einstein. The classification of the compact homogeneous quaternionic manifolds is precisely given by the Wolf spaces $\left[\overline{6}_{1}^{6}, 14 \overline{1}\right]$. Hence the $k=1$ instanton moduli spaces are characterized by the Wolf series. Quaternionic spaces also appear in the coupling of four-dimensional $\mathcal{N}=2$ hypermultiplets to supergravity [1대] instantons later.

For arbitrary instanton number $k$, one can not construct the instanton solution explicitly. However, using the ADHM formalism (which extensively uses a quaternionic notation), it is possible to write down the solutions in an implicit way [i]. The moduli space, denoted by $\mathcal{M}_{k}(G)$, has dimension $4 k N, 4 k(N-2)$ and $4 k(N+1)$ for gauge groups $S U(N), S O(N)$ and $S p(N)$ respectively. It is known to be a hyper-Kähler manifold of a special kind [i hyper-Kähler manifolds [1] ${ }_{1}^{1}$ ]', because it has the following properties:

- The metric on the moduli space, $g$, allows for a hyper-Kähler potential, $\chi$, which satisfies, in local coordinates,

$$
g_{A B}=D_{A} \partial_{B} \chi .
$$




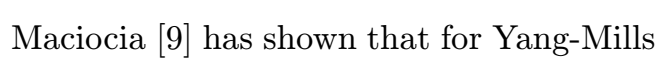
instantons,

$$
\chi=\frac{1}{16 \pi^{2}} \int \mathrm{d}^{4} x|x|^{2} \operatorname{Tr} F_{\mu \nu} F_{\mu \nu},
$$

and for $k=1$, one can explicitly compute this potential, $\chi=2 \rho^{2}+\left|x_{0}\right|^{2}$.

- The derivative $\chi_{A}=\partial_{A} \chi$ is a homothetic Killing vector, and there is an $S U(2)$ isometry, generated by

$$
\vec{k}^{A}=\vec{J}_{B}^{A} \chi^{B}
$$

where $\vec{J}$ are the three complex structures of the hyper-Kähler manifold. This $S U(2)$ isometry rotates the complex structures. Fourdimensional hyper-Kähler spaces with such an $S U(2)$ isometry are classified. They are either flat space, Taub-Nut, or the AtiyahHitchin manifold. Only flat space allows for a homothetic Killing vector.

- One can factor out the center of mass coordinates of the $k$-instanton solution, such that

$$
\mathcal{M}_{k}=\mathbf{R}^{4} \times \mathcal{M}_{k}^{0}
$$

Here $\mathcal{M}_{k}^{0}$ is the reduced instanton moduli space. It is again a special hyper-Kähler manifold [1] 1']. Spaces with a homothety can always be described as a cone. One can choose $\chi$ as one of the coordinates, and denote the remaining coordinates by $x^{\hat{A}}$. The line element can then be written in the form

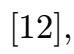

$$
\mathrm{d} s^{2}=\frac{\mathrm{d} \chi^{2}}{2 \chi}+\chi h_{\hat{A} \hat{B}}(x) \mathrm{d} x^{\hat{A}} \mathrm{~d} x^{\hat{B}},
$$

where the $x^{\hat{A}}$ are the coordinates associated with the hypersurface $\chi=$ constant. It is known that this hypersurface must be a 3-Sasakian space and the hyper-Kähler space is therefore a cone over the 3 -Sasakian metric. For a recent review on these spaces, see [1는.

For $k=1$ one can see the cone structure in the reduced moduli space metrics $\left(\begin{array}{l}1 \\ 1\end{array}\right.$ and ( $\left(\overline{1}_{1}^{\prime}\right)$. Indeed, the hyper-Kähler potential is, up to normalization, just the size of the instanton, $\chi=\rho^{2}$. At $\rho=0$, there is a conical singularity, corresponding to zero size instantons.

- It turns out that 3-Sasakian spaces are $S p(1)$ fibrations over quaternionic Kähler manifolds $\mathbf{Q}\left[\mathbf{1} 0_{1}^{\prime}\right]$. Hence one can write

$$
\mathcal{M}_{k}^{0}=\mathbf{R}^{+} \times\left[S p(1) \times{ }_{f} \mathbf{Q}_{k}\right],
$$

where $\mathbf{R}^{+}$is parametrized by the cone variable $\chi$, and the subscript $f$ denotes the non-trivial fibration. This structure is indeed present in the above discussed examples. For $k=1, \mathbf{Q}_{1}$ are precisely the Wolf spaces, which are the only compact homogeneous quaternionic manifolds. For higher instanton number, $\mathbf{Q}_{k}$ will in general not be homogeneous anymore, and it will contain singularities. It would be interesting to study its properties.

In an apparently different context, special hyper-Kähler manifolds also appear as target spaces for four-dimensional (but also in five or six dimensions ${ }^{2}$ ) superconformal hypermultiplets, as was discussed in detail in $\left[1 \overline{6}_{1}^{\prime}, \overline{1} \overline{7}_{1}^{\prime}, \overline{1} \overline{8}_{1}^{\prime \prime}\right]$, see also [199']. There, the implications of rigid $\mathcal{N}=2 \mathrm{su}-$ perconformal invariance on sigma models were analyzed, and the constraints on the hyper-Kähler manifold are exactly the ones discussed above. The corresponding quaternionic space then appears if one would couple the hypermultiplets to supergravity via the superconformal tensor calculus [201]. This raises the question if the instanton moduli space naturally appears as the target space of some sigma model theory. This is in-

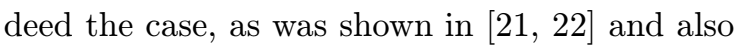
in [23in. There, instantons are described in terms of $\mathrm{Dp}-\mathrm{D}(\mathrm{p}+4)$ brane configurations, and the instanton moduli space coincides with the vacuum moduli space of the corresponding worldvolume theory of the $\mathrm{Dp}-\mathrm{D}(\mathrm{p}+4)$ system.

To make this correspondence more clear, we should consider instantons in $\mathcal{N}=4$ SYM theory at the conformal point (i.e., not spontaneously broken), and for simplicity we choose gauge group

\footnotetext{
${ }^{2}$ For two-dimensional sigma models on group manifolds, with eight supercharges, one also recovers the Wolf spaces [25].
} 
$S U(N)$ and instanton number $k=1$. On top of the $4 N$ bosonic zero modes, we have $8 N$ fermionic zero modes, each one corresponding to a solution of the Dirac equation. The Grassmannian collective coordinates (GCC) are denoted by

$$
\xi_{\alpha}^{M}, \bar{\eta}^{M \dot{\alpha}}, \mu_{i}^{M}, \bar{\mu}^{M i},
$$

see for instance $S U(4)_{R}$ symmetry index, $\alpha, \dot{\alpha}=1,2$ are twocomponent spinor indices, and $i=1, \ldots, N-2$ is related to the color index. The first sixteen of these GCC, $\xi^{M}$ and $\bar{\eta}^{M}$ are related to supersymmetry and superconformal supersymmetry, i.e., their zero modes can be obtained by acting with the super(conformal-) symmetry transformations on the bosonic instanton configuration. Only half of the symmetries are broken, so for $\mathcal{N}=4$ we get $8+8=16$ fermionic zero modes.

In instanton calculations, one is interested in the instanton action. This is obtained by plugging in the instanton configuration (which in $\mathcal{N}=$ $4 \mathrm{SYM}$ is only an approximate solution to the field equations) into the SYM action and integrating over four-dimensional space. The resulting action only depends on the collective coordinates, and for $k=1, S U(N)$ SYM, it is

$$
S_{\mathrm{inst}}=-\frac{\pi^{2}}{4 g^{2} \rho^{2}} \epsilon_{M N P Q} \mu_{i}^{M} \bar{\mu}^{N i} \mu_{j}^{P} \bar{\mu}^{Q j}
$$

where $g^{2}$ is the YM coupling constant.

The remaining of this paper is to understand this result in terms of superconformal hypermultiplet sigma models, and to point out the relation with the quaternionic geometry described above. All the collective coordinates are organized in hypermultiplets, and we have $N$ of them. Two hypermultiplets are special, they comprise the eight scalars $x_{0}^{\mu}, \rho, \vec{\theta}$ and fermions $\xi^{M}, \bar{\eta}^{M}$. This "universal" sector appears in every SYM theory, and is independent of the gauge group. The fermions of the universal sector will not appear in the instanton action, since they are protected by super(conformal-)symmetry. The other hypermultiplets contain the quaternionic gauge orientation zero modes $\theta_{i}^{M}$ and fermions $\mu_{i}^{M}, \bar{\mu}^{M i}$. The latter correspond to fermionic zero modes which can not be obtained by acting with fermionic symmetry transformations on the bosonic instan- ton configuration. Hence one might expect them in the instanton action, as $\left(\overline{1}_{1}^{\prime} \overline{9}_{1}^{\prime}\right)$ indeed confirms.

The instanton moduli space as a Higgs branch was first discovered in the D5-D9 system in type I string theory [21]]. The corresponding worldvolume theory on the Higgs branch is a sigma model in six dimensions with eight rigid supercharges. The general action for such a system contains kinetic terms for the scalars and fermions, and a four-fermi term coupled to the Riemann tensor of the $4 n$-dimensional hyper-Kähler target space $[\overline{2} \overline{6} \overline{-}]$,

$$
S_{4-\text { fermi }} \propto R_{a b c d} \epsilon_{M N P Q} \psi^{M a} \psi^{N b} \psi^{P c} \psi^{Q d} .
$$

Here $\psi^{M}$ is a six-dimensional $S U(4) \approx S O(6)$ symplectic Majorana spinor, $\epsilon_{M N P Q}$ is the totally antisymmetric $S U(4)$ invariant tensor, $a=$ $1, \ldots, 2 n$ is the $S p(n)$ index, and $R_{a b c d}$ is the totally symmetric $S p(n)$ curvature of the hyperKähler target space, which has holonomy group contained in $S p(n)$, see $\left[\overline{1} \overline{5}_{1}, \overline{2} \overline{2}\right]$ for more details. The instanton action in (i1 $\left.\overline{9}_{1}^{\prime}\right)$ can be compared with the system in (120) by dimensional reduction to zero dimensions. In terms of string theory, we are then describing the $\mathrm{D}(-1)$-D3 system, which is well known to represent a fourdimensional SYM instanton [2] $\left.\overline{2}_{1}^{\prime}\right]$. After dimensional reduction, all kinetic terms have disappeared and one is left with only the Riemann tensor as in $\left(20_{1}^{\prime}\right)$. Applied to the $k=1 S U(N)$ instanton model, we have $n=N$, and the $8 N$ fermions $\psi^{M a}$ comprise $\xi_{\alpha}^{M}, \bar{\eta}^{M \dot{\alpha}}, \mu_{i}^{M}, \bar{\mu}^{M i}$. Now, because the $\xi$ and $\bar{\eta}$ zero modes are protected by super(conformal-)symmetry, they cannot appear in the instanton action. So the only surviving components are the ones where the Riemann tensor has its indices in the subspace $S p(N-$ 2) $\subset S p(N)$. This is in accord with the general structure presented above. First one factors out the centre of mass coordinates, such that the reduced instanton moduli space has holonomy contained in $S p(N-1)$. As was shown in "1] $\overline{6}^{\prime}$, the $S p(N-1)$ curvature of this special hyperKähler space has a quaternionic zero eigenvector. This corresponds to the fact that the superconformal zero modes do not appear in the instanton action. Secondly, this reduced moduli space is a cone over an $S p(1)$ fibration of a $4(N-2)$ - 
dimensional quaternionic manifold, with holonomy group contained in $S p(1) S p(N-2)$. Its coordinates are given by $q_{i}^{M}$ and we expect that it is precisely the $S p(N-2)$ curvature which couples to the four-fermi term containing the $\mu_{i}^{M}$ 's. For $k=1$, one can compue the Riemann tensor of the instanton moduli space explicitly, it is essentially determined by the Riemann tensor of the corresponding Wolf space. Since these spaces are symmetric, there is a basis of coordinates in which all components of the curvature tensor (with two upper and two lower indices) are constant and determined by the structure constants of the coset space. This is consistent with (19.9.1), although the details of the computations remain to be worked out. We hope to come back to this in the future.

To conclude, we believe that the relation between instanton moduli spaces and quaternionic geometry is worth pursuing.

\section{Acknowledgments}

I would like to thank the organizers, in particular Bernard Julia, for inviting me to the conference, and for the warm and pleasant atmosphere during that week. I also acknowledge discussions with Tim Hollowood, V. V. Khoze and M. Trigiante.

\section{References}

[1] A. Belavin, A. Polyakov, A. Schwartz and Y. Tyupkin, Phys. Lett. B59 (1975) 85.

[2] G. 't Hooft, Phys. Rev. D14 (1976) 3432.

[3] M. Atiyah, Geometry of Yang-Mills Fields, Ann. Scuola Norm. Pisa, 1979.

[4] C. Bernard, Phys. Rev. D19 (1979) 3013.

[5] A. Belitsky, S. Vandoren and P. van Nieuwenhuizen, Class. Quantum. Grav. 17 (2000) 1, hep-th/0004186.

[6] J. Wolf, J. Math. Mech. 14 (1965) 1033.

[7] C. Bernard, N. Christ, A. Guth and E. Weinberg, Phys. Rev. D16 (1977) 2976.

[8] M. Atiyah, V. Drinfeld, N. Hitchin and Y. Manin, Phys. Lett. A65 (1978) 185.

[9] A. Maciocia, Commun. Math. Phys. 135 (1991) 467.
[10] A. Swann, Math. Ann. 289 (1991) 421.

[11] C. Boyer and B. Mann, Proc. of Symp. in Pure Math. 54, part 2 (1993) 45.

[12] G.W. Gibbons and P. Rychenkova, Cones, tri-Sasakian structures and superconformal invariance, Phys. Lett. B443 (1998) 138, hep-th/9809158.

[13] C.P. Boyer and K. Galicki, 3-Sasakian Manifolds, to appear in Essays on Einstein Manifolds, M. Wang and C. LeBrun, eds, hep-th/9810250.

[14] D.V. Alekseevskii, Funct. Anal. Appl. 2 (1968) 97.

[15] J. Bagger and E. Witten, Nucl. Phys. B222 (1983) 1.

[16] B. de Wit, B. Kleijn and S. Vandoren, Nucl. Phys. B568 (2000) 475, hep-th/9909228.

[17] B. de Wit, B. Kleijn and S. Vandoren, in Supersymmetries and Quantum Symmetries", proc. Int. Sem. Dubna (1997), eds. J. Wess and E.A. Ivanov, Lecture Notes in Physics, Vol. 524 (Springer, 1999), p. 37, hep-th/9808160.

[18] B. de Wit, M. Roček and S. Vandoren, to appear.

[19] K. Galicki, Class. Quantum Grav. 9 (1992) 27.

[20] B. de WIt, J.W. van Holten and A. Van Proeyen, Nucl. Phys. B167 (1980) 186.

B. de Wit, P. Lauwers and A. Van Proeyen, Nucl. Phys. B255 (1985) 569.

[21] E. Witten, Nucl. Phys. B460 (1996) 541, hep-th/9511030.

[22] M. Douglas, In "Cargese 1997, Strings, Branes and dualities", 267, hep-th/9512077; J. Geom. Phys. 28 (1998) 255, hep-th/9604198.

[23] N. Dorey, T. Hollowood, V. Khoze, M. Mattis and S. Vandoren, Nucl. Phys. B552 (1999) 88, hep-th/9901128.

[24] N. Dorey, V. Khoze, M. Mattis and S. Vandoren, Phys. Lett. B442 (1998) 145, hep-th/9808157.

[25] A. Sevrin, Ph. Spindel, W. Troost and A. Van Proeyen, Nucl. Phys. B308 (1988) 662, Nucl. Phys. B311 (1988) 465.

[26] G. Sierra and P. Townsend, Nucl. Phys. B223 (1984) 289.

[27] J. De Jaegher, B. de Wit, B. Kleijn and S. Vandoren, Nucl. Phys. B514 (1998) 553, hep-th/9707262. 